\title{
[ESCRIBIENDO PARA LA ESCENA. LAS ANOTACIONES DE la compañía de luna para El estreno de "Don Álvaro de Luna", de Gil y Zárate (1840)]
}

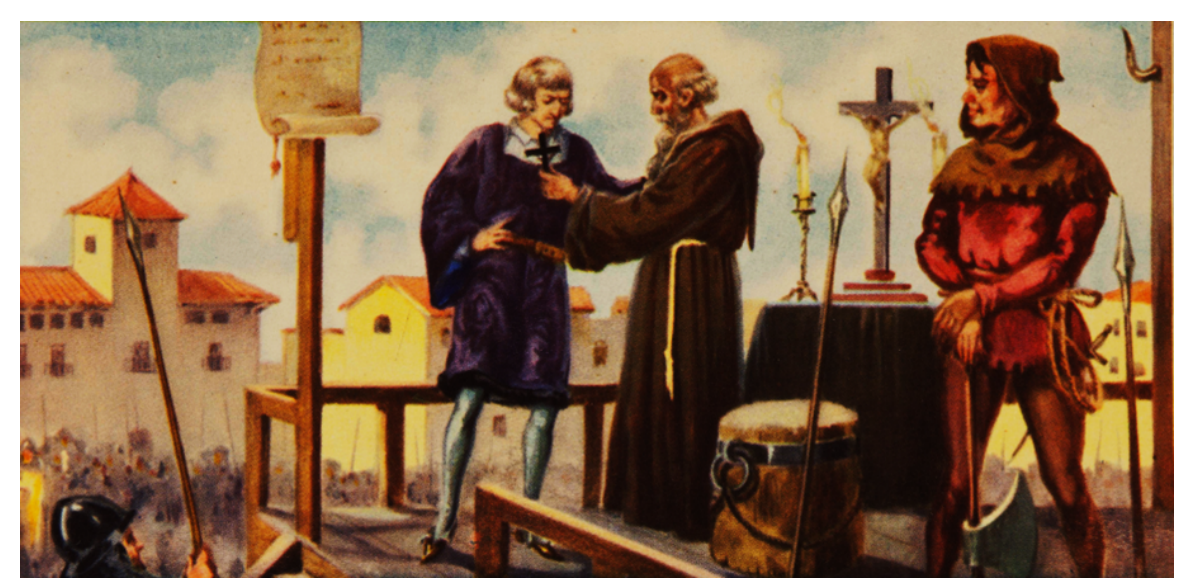

E desque fue llegado a él, descabalgó de la mula e subió sin empacho alguno por los escalones del tal cadalso; e después que fue subido encima e se vido allí adonde la alombra estaba tendida, tomó un sombrero que traía en su cabeza e echolo a uno de aquellos pajes suyos, el que ya diximos que se llamaba Morales. (Crónica de don Álvaro de Luna, Madrid: Sancha, I784, p. 380).

Antes de entregarse a la muerte, da su anillo, como último recuerdo, a su fiel escudero Morales. Fija en Dios su pensamiento, se arrodilla sobre un negro cojín e inclina la cabeza, sobre la cual vuela el hacha del verdugo. Víctima de la intriga y de la ingratitud muere así el que fue famoso y brillante condestable de Castilla, don Álvaro de Luna (Cromos culturales. Don Álvaro de Luna, Barcelona: Barsal, I930-1940, tarjeta X). 



\title{
ESCRIBIENDO PARA LA ESCENA. LAS ANOTACIONES DE
} LA COMPAÑía DE Luna PARA EL ESTRENO DE "DON

\author{
Álvaro de LunA", DE Gil y ZÁrate (1840) \\ Ma CARMen Rodríguez Lorenzo \\ Universidade de Vigo
}

Title: Writing for the scene. The annotations of the company of Luna for Don Álvaro de Luna's premiere, of Gil y Zárate (I 840)

Abstract: Of Antonio Gil y Zárate's drama Don Álvaro de Luna (I 840) have remained three manuscript notes used for the putting in scene during his premiere and guarded today by the Municipal Library of Madrid, which information helps us to know on his representation. In this work there are analyzed the information that contribute these notes and the differences between these and the printed edition.

Key words: Álvaro de Luna. Gil y Zárate. Manuscript notes. Theatre. Romanticism. Romantic Drama.

En febrero de 1840, en el madrileño teatro del Príncipe, la compañía del actor Luna lleva a escena el drama histórico en cinco actos Don Álvaro de Luna, de Antonio Gil y Zárate, ${ }^{\mathrm{I}}$, que sube a las tablas las cuestiones históricas y las disputas personales que acompañaron al condestable en los días previos a su ajusticiamiento. Durante la temporada 39-40 el Príncipe estaba dirigido por el consorcio de actores ${ }^{2}$ que dos temporadas atrás se había hecho cargo de esta sala y de La Cruz. En la temporada 36-37, ante la situación precaria por que atraviesa el mundo del teatro debido a los problemas económicos del momento, se intenta hacer reformas que mejoren el aspecto de las salas y se contrata a un nuevo censor, Sancha, cuya dimisión a finales de I 837 provoca, a su vez, desavenencias entre el Ayuntamiento y la Comisión de Teatros a propósito de quién debe ocupar el cargo. Todos estos

\footnotetext{
${ }^{1}$ Este trabajo se inserta en el ámbito del proyecto de investigación FFI20 5-64IO7-P (MINECO-FEDER, UE).

${ }^{2} \mathrm{El}$ consorcio lo formaban los actores José García Luna, que era la cabeza visible, José Guzmán y Carlos Latorre. Se hicieron cargo del Príncipe en la temporada 36-37 por un período de tres años (Ribao I 998: 164-165).
} 
problemas repercuten en la temporada 39-40, ya que las dificultades persisten, haciendo que el número de obras originales llevadas a escena disminuya y que los decorados y los elencos sean insuficientes para poder reflejar el esplendor previsto en la escritura dramática de los textos. El consorcio no puede hacerse cargo de todas las deudas de las salas y el Ayuntamiento, finalmente, propone la supresión del cargo de censor, que se hará efectiva en enero de I840 (Ribao, I998: I64-175).

A pesar de todo, el Príncipe sigue abriendo sus puertas con normalidad y la compañía dirigida por Luna lleva a las tablas algunas obras relevantes. Entre las piezas representadas se encuentra el drama de Antonio Gil y Zárate Don Álvaro de Luna, que se estrena en febrero de i 840 y se mantiene en cartel doce días. ${ }^{3}$

El drama cuenta con dos ediciones impresas (Madrid, Yenes: i 840; París: Baudry, i 850), pero también se conservan tres manuscritos apógrafos que coinciden con los apuntes utilizados para la representación por la compañía, en los que se pueden encontrar indicaciones sobre decorado, entradas y salidas, entonación..., información toda ella inexistente en las ediciones de la obra (Ribao, I 999; Aguerri Martínez, 2007; González Martínez, 2014) y a la que voy a referirme en las páginas que siguen.

Los apuntes de Don Álvaro de Luna se encuentran en la Biblioteca Municipal de Madrid. Se trata de tres cuadernos cosidos, aparentemente, a mano y formando pliegos con diverso número de páginas, correspondientes cada uno a un acto de la obra. Cada apunte tiene una función. El primero es una copia en limpio en la que no aparecen correcciones y puede ser el resultado de transcribir, sin enmiendas ni tachaduras, la obra después de los cambios introducidos por la compañía en los demás apuntes. El segundo indica entradas y salidas de actores y sus nombres o los lugares en donde

${ }^{3}$ Don Álvaro de Luna se representa durante varios días alternos del mes de febrero: I-4, 7-8, I I-16. La repercusión en prensa del estreno es importante, tanto por la naturaleza de la obra como por la categoría de su autor. Para todo ello vid. Rodríguez Lorenzo (2017). 
deben situarse para salir. El tercero es muy parecido al segundo, pero en él se da mayor relevancia a los datos sobre decorados y efectos de luz (Ribao, I 999: 70-72). En el caso que nos compete, me referiré a ellos como MB (primer apunte), MA y MC, respectivamente. Describo a continuación cada uno de ellos.

El apunte MA se ha conservado completo y la Biblioteca Municipal de Madrid da la siguiente información sobre la composición de los actos: el primero está integrado por treinta y una hojas; en el segundo encontramos trece pliegos de dos hojas, en el tercero y quinto hallamos doce pliegos de dos hojas y en el cuarto tenemos once también de dos hojas. La portada del primer acto de este manuscrito es la más completa e incluye la catalogación de la biblioteca (L. I r. N.36), título de la obra, año, número de acto y una rúbrica, que puede coincidir con la del apuntador. En el resto de actos figura en la portada la catalogación, el título, el número de acto y la rúbrica. Las anotaciones de este apunte marcan las entradas y salidas de actores, el lugar desde donde lo hacen y también, aunque en menor medida, se pueden observar algunas indicaciones con respecto a la entonación.

El manuscrito MB no está completo, ya que le falta el quinto acto. $\mathrm{Al}$ igual que en MA, la Biblioteca ${ }^{4}$ indica que el primer acto se compone de dieciséis pliegos de dos hojas; el segundo consta de quince, el tercero cuenta con doce pliegos y el cuarto tiene diez. La portada del acto I sigue la misma estructura que en MA, pero en MB se anota que es el primer apunte, hay una rúbrica distinta ${ }^{5}$ y la signatura $\mathrm{I}-25-4$. El resto de las portadas coinciden con MA salvo en la rúbrica. Como he comentado anteriormente, este apunte es el más próximo, desde el punto de vista textual, a la edición, pero en el

${ }^{4}$ Cuando aluda a la "Biblioteca" haré referencia a la Biblioteca Municipal de Madrid, que conserva una rica colección teatral y los valiosos materiales manuscritos relacionados con la puesta en escena de los dramas que las compañías contratadas por el Concejo llevan a escena durante varios siglos. Para todo ello vid. Aguerri (2007).

${ }^{5} \mathrm{~L}$ a rúbrica que aparece en la portada del acto I del apunte MB es la misma que aparece al final de cada acto de este mismo apunte. 
caso de Don Álvaro de Luna se pueden encontrar diferencias entre el texto literario del impreso y el de los manuscritos. Veamos un ejemplo:

MA y MB: "Pacheco: No importa, que hoy entretenidos, / con tan soberbia función, [...]". (I, I).

Edición: "Pacheco: Las auras de este jardín / se llevarán nuestra voz / demás que hoy entretenidos / con tan soberbia función, [...]”.(I, I, I840: 3; I850: 2OI).

Como se puede ver, en MA y MB no aparecen los versos "las auras de este jardín/ se llevarán nuestra voz" y se ofrece una solución diferente para el siguiente. La edición, al tratarse de una impresión posterior, añade estos dos versos y pone de relieve que el producto destinado a la lectura y el que se representa son independientes, dirigidos a dos receptores distintos que, por consiguiente, no tienen por qué coincidir, como pasa, de hecho, en el desenlace, al que más adelante me referiré.

Podemos apreciar en este apunte una única referencia a la puesta en escena, que alude a la iluminación y a la entrada de actores: al inicio del acto III, tras la alusión a los personajes que entran en escena (Pacheco y Vivero), $\mathrm{MB}$ anota al margen, con una caligrafía diferente a la de los versos y las acotaciones, la indicación "Oscuro", así como esta marca,

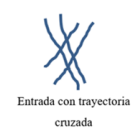

con la que se señala el movimiento actoral describiendo trayectorias cruzadas (Ribao, 1999).

Pese a que aparece alguna pequeña enmienda en él, se aprecia en MB una letra más cuidada que en MA y MC, debido a que este cuadernillo es el punto de partida del texto que se lleva a la imprenta para su edición.

Del tercer y último apunte, MC, solo se conservan los actos cuarto y quinto. Siguiendo el mismo esquema que en los anteriores, la Biblioteca 
indica que al acto cuarto consta de diez pliegos de dos hojas y el quinto de once. Las portadas de ambos actos siguen el modelo de MA, en este caso sin rúbrica alguna. Tenemos constancia de que es el apunte que se envió a la Comisión, pues en el final de la obra aparece su aprobación explícita: "Puede hacerse. Por la comisión. Sancha”. El hecho de que en este apunte figure el consentimiento para la puesta en escena corrobora que el drama no contaba todavía con edición impresa en el momento de prepararse su montaje, pues de ser así ya habría obtenido la necesaria aprobación previa a su paso por las prensas.

MC recoge datos sobre entrada y salida de actores, decorados, acciones que no son visibles para el público, alguna de iluminación... No se encuentran, sin embargo, referencias a la entonación que deben realizar los actores. A continuación reproduzco algunos ejemplos de las diferentes indicaciones de este manuscrito:

- Barba, Castañón, Lombía, Ramírez, Spuntoni, Reyes, Barja con caballeros. Pajes aparecen (IV, I).

- Lombía izquierda arriba (IV, I).

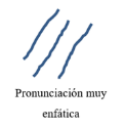

- Entrada en escena de comitiva $(\mathrm{V}, 6)$ y pronunciación muy enfática (IV, 2; V, 8).

- Martínez foro derecha $(\mathrm{IV}, 2)$.

- Álvaro foro derecha (IV, 2).

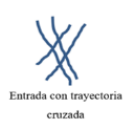

3 y 5$)$.

- Entrada en escena con trayectoria cruzada (IV, 3, 6 y 7; V,

- Galán fondo derecha (IV, 4).

- Lombía, caballeros, ocho guardias. Foro derecha (IV, 6).

- Mesa, silla, reloj de arena. Galán y bravo aparecen (V, I).

- Reloj da las dos (V, I). 
- Las dos. Escena fuera de la vista del espectador (V, I).

- Barba, Bravo. Derecha puerta $(\mathrm{V}, 2)$.

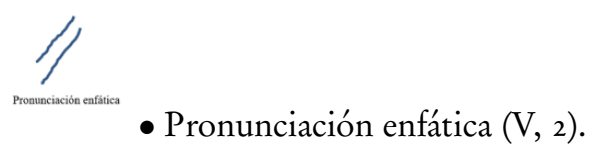

- Lombía derecha puerta $(\mathrm{V}, 3)$.

- Don Álvaro, Bravo, derecha puerta $(\mathrm{V}, 3)$.

- Lledó, alguaciles, dos alcaldes, 8 soldados, derecha puerta $(\mathrm{V}, 4)$.

- Los oficiales entran. Galán y dos frailes. Y al mismo tiempo criados $(\mathrm{V}, 4)$.

- Clarín con (ilegible). Lledó en entrando. Pregón dos veces. Dentro derecha $(\mathrm{V}, 6)$.

- Dama en entrando con pliego $(\mathrm{V}, 6)$.

- Lombía derecha puerta $(\mathrm{V}, 6)$.

- Reloj que da las tres. Foro izquierda $(\mathrm{V}, 6)$.

- Cadalso. Pueblo. Guardias p.do al fondo y los oficiales entran (V, 6).
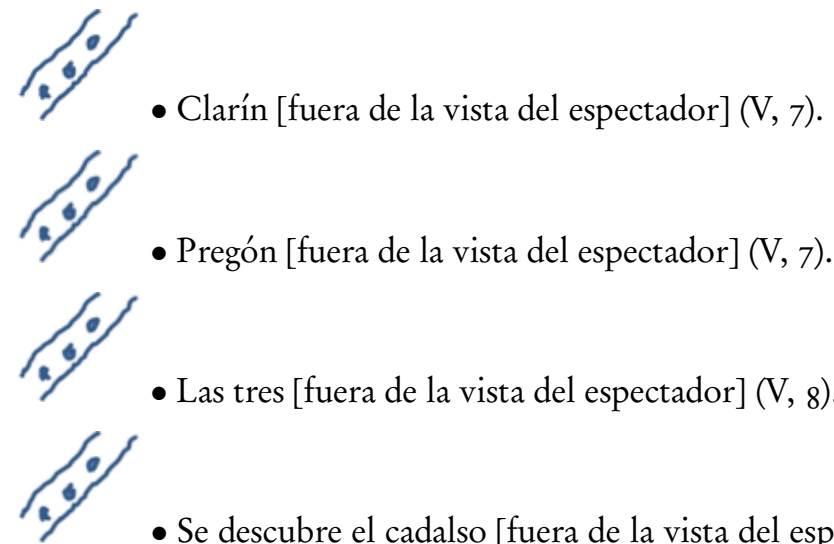

8).

- Se descubre el cadalso [fuera de la vista del espectador] (V,

También en MC se pueden encontrar algunas pequeñas diferencias en el texto literario con respecto al impreso, tal y como ocurría en MA. Reproduzco, a continuación, algunos ejemplos: ${ }^{6}$

\footnotetext{
${ }^{6}$ Señalo en cursivas las diferencias entre las soluciones de los testimonios
} 
Escribiendo para la escena. Las anotaciones de la compañía de Luna para el estreno

MC:

SANTILLANA

De amor he querido

en cántiga breve

la voz definir,

mas fue vana empresa;

dichoso no he sido.

REY

Teneisla.

SANTILLANA

Sí, tengo.

REY

Pues quiérola oír.

(IV, I)

Edición (I 840 y I 850 ):

SANTILLANA

De amor he querido

en cántiga breve

la voz definir,

mas fue vana empresa;

dichoso no he sido.

REY

¿Teneisla?

Sí, tengo.

REY

Pues quiérola oír.

(IV, I, I 840: 75; I 850: 226-227).

MC:

SANTILLANA

E yo también sin ventura,

en vos buscando placer,

fallé solo padecer

cuita en lugar de folgura.

(IV, I).

Edición (I 840 y I 850 ): 


\section{SANTILLANA}

E yo también sin ventura,

en vos buscando placer,

fallé solo padescer

cuita en lugar de folgura.

(IV, I, I 840: 75-76; I 850: 227).

MC:

\section{SANTILLANA}

[...] amor preferencia quiere decir, es cierto, pues tiene el que elige voluntad advierto y no hay en quien ama jamás voluntad. (IV, I).

\section{Edición (I 840 y I 850 ):}

SANTILLANA

[...] amor preferencia quiere decir, es cierto, mas siempre no elige para ella, pues tiene el que elige voluntad advierto y no hay en quien ama jamás voluntad.

(IV, I, I 840: 76; I 850: 227).

MC:

REY

No..., no temas.

Allí está. Vive tranquila...

Hora se apartó de mí.

ELVIRA

¿Le habéis visto?

REY

Sí, hija mía.

ELVIRA

Luego está perdonado.

REY

¡Perdonado!

$(\mathrm{V}, 4)$.

Edición (I 840 y I 850 ): 
REY

No..., no temas.

Allí está. Vive tranquila...

Hora se apartó de mí.

ELVIRA

¿Le habéis visto?

REY

Sí, hija mía.

ELVIRA

¿Luego perdonado está?

REY

¡Perdonado!

(V, 4, I 840: 102; I 850: 236).

Sin embargo, hay una variante en los versos finales del drama sobre la que es preciso llamar la atención. Los últimos versos de la obra, idénticos en MA y en MC, aparecen tachados en este último, probablemente por indicación de la Comisión, que los sustituye (la letra de la interpolación es diferente) por una solución similar, pero en la que se suprime la muerte de la protagonista, cuyo destino queda en suspenso. Esta variante es la que se imprime, pero no la que se representa, porque los versos en cuestión no se eliminan de MA, sino que se mantienen, circunstancia esta que en unos apuntes para la puesta en escena solo tiene sentido si corresponden al parlamento de algún actor:

Edición (I 840: I09; I 850: 259) y MC (s. p.):

ACTO V

Escena 8

Elvira: Sí, sí... ¡Perdón! ¡Perdón... !Mirad... Teneos.

(Destúñiga y Morales corren a la ventana del fondo y la abren de par en par. Se ve una plaza y el cadalso en que está ya Don Álvaro degollado. Elvira corre hacia la ventana gritando y mostrando el perdón, pero al ver muerto a su padre da un grito y cae desmayada en los brazos de Destúñiga y Morales).

Destúñiga: ¡Cielos...! ¡No es tiempo ya! 
Elvira: ¡Jesús mil veces!

MA (s. p.):

ACTO V

Escena 8

Elvira: Sí, sí... ¡Perdón! ¡Perdón. ... Mirad.... Teneos.

¡Jesús!

Destúñiga: ¡Ah! ¡Muere!

El texto espectacular apenas presenta variaciones entre los apuntes y la edición, salvo las indicaciones dirigidas a los actores a las que antes me he referido, ya que el texto impreso carece de las marcas de entradas y salidas de personajes, de los avisos a los actores y de telón, de entonación... necesarias para la compañía. Las diferencias en las acotaciones de los manuscritos y ediciones son menores y afectan, fundamentalmente, al estilo y a la redacción de las mismas, como podemos observar en estos ejemplos:

Edición (I 840: 8; I 850: 203): "Sale Elvira pensativa llevando en la mano una banda" (I, 2).

MA (s. p.) y MB (s. p.):“Lleva en la mano una banda” (I, 2).

Edición (I 840: 29; I 850: 2 I0): “Sale muy agitado y luego se sienta”. (II, I).

MA (s.p.): Sin acotación.

MB (s.p.): "Llega muy agitado y luego se sienta” (II, I).

Edición (I 840: 55; I 850 220): “Señalando la de la derecha” (III, I).

MA (s.p.): "Señala derecha” (III, I).

Edición (I 840: 76; I 850: 227): “Ruido dentro de gentes” (IV, I).

MA (s.p.), MB (s.p.) y MC (s.p.): “Ruido dentro” (IV, I).

Edición (I 840: 92; I 850: 232): "Se arrodilla delante de Don Álvaro y le besa la mano. Don Álvaro se despierta” (V, I).

MA (s.p.) y MC (s.p.): "Se arrodilla y le besa la mano. Don Álvaro se despierta" $(\mathrm{V}, \mathrm{I})$. 
Desde el punto de vista formal, se podría decir que Gil y Zárate busca que en el texto destinado a la edición impresa las acotaciones no sean tan escuetas como en las copias utilizadas para la puesta en escena. Cabría deducir, pues, que el dramaturgo pensó un texto para el espectador asistente al teatro y otro para el público lector.

Las acotaciones, tanto de los manuscritos como de los impresos, son nominativas, para nombrar a los interlocutores; psicológicas, para saber los sentimientos o ideas de los personajes; corporales, que indican gestos o movimientos; espaciales, referidas al decorado, la iluminación y los accesorios; y sonoras, para indicar si hay ruidos o suena alguna música. Sin embargo, las acotaciones paraverbales de prosodia y entonación (Aguerri, 2007: 140I 4I) solo aparecen en los manuscritos, no pasan a las ediciones y, por tanto, sustraen al lector información relevante y muy relacionada con la grandilocuencia de la estética romántica a la que se adscribe el drama.

El dinamismo de la acción que se deriva de los cambios de espacios y decorados o de la constante entrada y salida de personajes es corroborado por los apuntes. Gracias a ellos podemos saber, por ejemplo, que cada actor suele salir a escena habitualmente por el mismo lugar, de modo que se crea una asociación visual entre espacio y personaje que refuerza la espectacularidad del drama. Este es el caso concreto de los protagonistas: Elvira (Teodora Lamadrid o "dama”, en el argot de la compañía) entra por la izquierda, Destúñiga (Alverá) por la derecha y don Álvaro (Luna o "galán”) por el foro, para que su avance hacia el proscenio le brinde el mayor espacio posible para mostrar su protagonismo:

MA (s.p.): "Teodora izquierda". (I, I).

MA (s.p.): "Alverá derecha”. (I, 3).

MA (s.p.): "Galán, Barba, Lombía, Castañón, Ramírez, Spuntoni, Barja, reyes, Saavedra y caballeros, Zafra, derecha arriba”. (I, s).

MA (s.p.): "Zafra foro izquierda. Teodora izquierda. Dentro foro izquierda”. (II, 3). 
MA (s.p.): “Alverá, Lombía, Castañón, Spuntoni, reyes, Barja, pajes, escuderos con pendones, caballeros foro derecha. Guzmán, pajes, caballeros”. (II, 6).

MA (s.n.): "Martínez y Alverá foro derecha”. (IV, 3).

MA (s.n.): “Teodora y Bravo puerta izquierda”. (V, 3).

MA (s.n.): “Teodora puerta segunda izquierda con pliego entrando”. (V, 7).

Además de todos los datos mencionados en la descripción de los apuntes, gracias a estos conocemos también el nombre de algunos de los actores que formaban parte del elenco que llevó a cabo la representación: José García Luna, Juan Lombía, Ildefonso Zafra, Teodora Lamadrid, Antonio Alverá, José Castañón, José Ramírez, Carlos Spuntoni, Barja, Felipe Reyes, Saavedra, José Guzmán, Ángel López, Antonio Cobos, Martínez, Bravo y Casanova. Aunque en ninguno de los tres apuntes se asignan los personajes a los actores, gracias a los avisos de entradas y salidas podemos deducir qué actores daban vida a los personajes principales: Pacheco: Lombía; Vivero: Zafra; don Álvaro: Luna; Estúñiga: Álverá; y Elvira: Teodora Lamadrid.

Como vemos, gracias a los apuntes podemos tener información sobre cómo se preparó la representación de Don Álvaro de Luna. El texto originariamente propuesto a la compañía por el dramaturgo es objeto de sutiles modificaciones, enmiendas y cambios que no proceden de una decisión autorial, sino de las necesidades o gustos de la compañía o de la censura. En algunos casos, como en el desenlace, los versos que escucha un asistente al teatro en el estreno y funciones siguientes no coincide con los que las ediciones recogen, lo que implica que un lector actual no conoce, en sentido estricto, el drama aplaudido en su momento, ni el reseñado por la crítica, sino solo una versión del mismo. La escena reescribe, en este sentido, la voluntad del dramaturgo para adaptarse a la especificidad de un género en cuya complejidad radica, precisamente, una de las claves de su riqueza. 
Escribiendo para la escena. Las anotaciones de la compañía de Luna para el estreno de "Don Álvaro de Luna", de Gil y Zárate (1840)

\section{BIBLIOGRAFÍA}

Aguerri Martínez, Asunción (2007), "La catalogación de los apuntes de teatro en la Biblioteca Histórica Municipal”, Revista General de Información y Documentación, I7, I, pp. 133-164.

Gil y Zárate, Antonio (i 840), Don Álvaro de Luna, Madrid: Yenes.

Gil y ZÁrate, Antonio (s. a.), Don Álvaro de Luna, MA, Biblioteca Municipal Histórica de Madrid.

Gil y ZÁrate, Antonio (s. a.), Don Álvaro de Luna, MB, Biblioteca Municipal Histórica de Madrid.

Gil y ZÁrate, Antonio (s. a.), Don Álvaro de Luna, MC, Biblioteca Municipal Histórica de Madrid.

GonzÁlez Martínez, Javier (20I4), "La autoría del apunte teatral El triunfo mayor de Ciro y la impresa Araspas y Pantea" Revista de Filología Española, XCIV, I, pp. I07- I 26.

Ribao Pereira, Montserrat ( I998), "Vicisitudes empresariales de los teatros de La Cruz y El Príncipe en el Madrid de la Regencia”, Boletín de la Biblioteca de Menéndez Pelayo, 74, pp. I 5 5-178.

Ribao Pereira, Montserrat (i999), "Acerca de los apuntes y sus posibilidades en el estudio del teatro romántico y español (1 835-1 845)”, España Contemporánea, I 2, 2, pp. 67-86.

Rodríguez Lorenzo, María del Carmen (2017), “Álvaro de Luna (1840), de Antonio Gil y Zárate, ante la prensa de su época”, Lectura y Signo, I 2, pp. 22 1-237.

recibido: septiembre de 2018

aceptado: noviembre de 2018 
